\title{
Family exposure and the impact of containment measures to children with coronavirus disease 2019 outside Hubei, China: a cross-sectional study
}

\author{
Shu Yang ${ }^{1,2 \#}$, Xixi Feng ${ }^{3 \#}$, Peipei $\mathrm{Du}^{2,3 \#}$, Daihai He ${ }^{4}$, Janne Estill ${ }^{5,6}$, Lin Yang ${ }^{7}$, Chuanbiao Wen ${ }^{1,2}$, Jiawei Luo $^{8}$, \\ Xiaohui Wang', Liqun Lu ${ }^{10}$, Tingting $\mathrm{Li}^{11}$, Xiujuan Tang ${ }^{12}$, Weiguo Li ${ }^{13}$, Hongmei $\mathrm{Xu}^{13}$, Enmei Liu ${ }^{13}$, \\ Peihua $\mathrm{Cao}^{14}$, Yaolong Chen ${ }^{15,16,17,18}$; on behalf of COVID-19 evidence-based research working group \\ ${ }^{1}$ College of Medical Information Engineering, Chengdu University of Traditional Chinese Medicine, Chengdu, China; ${ }^{2}$ Digital Institute of \\ Medicine, Chengdu University of Traditional Chinese Medicine, Chengdu, China; ${ }^{3}$ School of Public Health, Chengdu Medical College, Chengdu, \\ China; ${ }^{4}$ Department of Applied Mathematics, Hong Kong Polytechnic University, Hong Kong, China; ${ }^{5}$ Institute of Global Health, University of

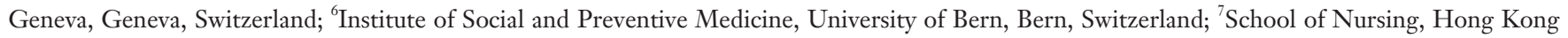 \\ Polytechnic University, Hong Kong, China; ${ }^{8}$ West China Biomedical Big Data Center, West China Clinical Medical College of Sichuan University, \\ Chengdu, China; 'School of Public Health, Lanzhou University, Lanzhou, China; ${ }^{10}$ Department of Pediatric, The First Affiliated Hospital of \\ Chengdu Medical College, Chengdu, China; ${ }^{11}$ School of Pharmacy, Chengdu Medical College, Chengdu, China; ${ }^{12}$ Shenzhen Center for Disease \\ Control and Prevention, Shenzhen, China; ${ }^{13}$ National Clinical Research Center for Child Health and Diseases, Children's Hospital of Chongqing \\ Medical University, Chongqing, China; ${ }^{14}$ Clinical Research Center, Zhujiang Hospital, Southern Medical University, Guangzhou, China; ${ }^{15}$ Evidence- \\ Based Medicine Center, School of Basic Medical Sciences, Lanzhou University, Lanzhou, China; ${ }^{16}$ WHO Collaborating Centre for Guideline \\ Implementation and Knowledge Translation, Lanzhou, China; ${ }^{17}$ GIN (Guidelines International Network) Asia, Lanzhou, China; ${ }^{18}$ Key Laboratory of \\ Evidence Based Medicine and Knowledge Translation of Gansu Province, Lanzhou University, Lanzhou, China \\ Contributions: (I) Conception and design: S Yang, X Feng, P Cao, Y Chen; (II) Administrative support: X Feng, P Du, L Lu, P Cao; (III) Provision \\ of study materials or patients: P Du, C Wen, J Luo, X Wang, L Lu; (IV) Collection and assembly of data: P Du, T Li, X Tang, W Li, H Xu, E Liu; \\ (V) Data analysis and interpretation: S Yang, X Feng, P Du, D He, J Estill, L Yang, P Cao, Y Chen; (VI) Manuscript writing: All authors; (VII) Final \\ approval of manuscript: All authors. \\ "These authors contributed equally to this work. \\ Correspondence to: Dr. Peihua Cao. 10/F, Clinical Research Center, Zhujiang Hospital, Southern Medical University, No.253 Gongyedadao Avenue, \\ Guangzhou, China. Email: cphcc@smu.edu.cn; Prof. Yaolong Chen. No. 199 Donggang West Road, Evidence-Based Medicine Center, School of \\ Basic Medical Sciences, Lanzhou University, Lanzhou, China. Email: chenyaolong@1zu.edu.cn.
}

Background: In response to the ongoing epidemic of coronavirus disease 2019 (COVID-19), China has carried out restrictive disease containment measures across the country.

Methods: In this cross-sectional study, we collected demographic and epidemiological data of 376 laboratory-confirmed cases of COVID-19 among children younger than 18 years of age. Using descriptive statistics and odds ratios, we described the odds of exposure outside the family after the implementation of control measures compared to before.

Results: Children diagnosed on or after February 4, 2020, had a lower odds of exposure to COVID-19 outside of the family compared to those diagnosed before February 3, 2020 (OR =0.594, 95\% CI: 0.391 to 0.904). In the stratified analysis, children aged 0 to 5 years had the lowest odds of exposure outside of the family (OR $=0.420,95 \% \mathrm{CI}: 0.196$ to 0.904 ) compared to the other age groups assessed.

Conclusions: Our study on the children infected with COVID-19 as well as their exposure within family provided evidence that the implementation of containment measures was effective in reducing the odds of exposure outside of the family, especially for preschool children. Continuation of these efforts, coupled with tailored prevention and health education messaging for younger aged children, may help to reduce the transmission of COVID-19 among children until other therapeutic interventions or vaccines are available.

Keywords: Children; containment measures; coronavirus; COVID-19; family exposure 
Submitted Jul 27, 2020. Accepted for publication Nov 13, 2020.

doi: $10.21037 /$ tp-20-214

View this article at: http://dx.doi.org/10.21037/tp-20-214

\section{Introduction}

An ongoing epidemic of coronavirus disease 2019 (COVID-19), caused by the severe acute respiratory syndrome coronavirus 2 (SARS-CoV-2), has spread throughout China and across the globe since December 2019 (1). As this a novel virus, no specific therapeutics or vaccines are yet available to prevent and treat COVID-19. Additionally, the beginning of the outbreak in China began immediately before Chinese Lunar New Year holiday, which often experiences substantial population movement, leading to an increased risk of transmission of the disease. Thus, the Chinese government needed to quickly implement unprecedented, large-scale containment measures (2). In the initial months of the epidemic, the government implemented strict travel bans and social distancing techniques, such as school closures, suspension of public markets, cancellation of public gatherings, working from home, and locking down entire cities or areas (3). Combining all these non-pharmaceutical public health interventions, China succeeded in curb outbreak trend of COVID-19. However, keeping a massive population sheltering in house may simultaneously elicit the risk of family exposure. A large number of clinical case reports indicate that alongside the implementation of containment measures, there have been increasing reports of familial clusters of COVID-19 infections, especially among children (4-7). In light of a retrospective study with 44,672 confirmed cases of COVID-19 in China, the proportion of confirmed case was $0.9 \%$ and $1.2 \%$ for the age group of $0-9$ and 10-19 years, respectively. However, another retrospective study including 2,143 pediatric patients with COVID-19 reported to the Chinese Center for Disease Control and Prevention from Jan 16 to Feb 8 in China demonstrated that $5.6 \%$ of 2,143 children had severe disease while $0.6 \%$ children developed respiratory or multiorgan failure or acute respiratory distress syndrome (ARDS), and revealed that young children, particularly infants, were vulnerable to SARS-CoV-2 infection (8). Thus, in this study, we described the epidemiologic and demographic features of COVID-19 patients under 18 years of age and evaluated the effects of containment measures on family exposure of COVID-19 infection among children of different age groups outside Hubei in China. We present the following article in accordance with the STROBE reporting checklist (available at http://dx.doi.org/10.21037/tp-20-214).

\section{Methods}

\section{Data sources}

The ethical approval or individual consent was not applicable. Data were analyzed at aggregate level and no participants were contacted. The study was performed in accordance with the Declaration of Helsinki (2013 revision). We collected data of reported confirmed COVID-19 patients under 18 years of age before Feb 14, 2020. The data on the number of COVID-19 diagnosed patients under 18 years of age were extracted from the official websites of China Health Commission, provincial and municipal Health Commissions from 30 provinces outside Hubei in mainland China, together with Hong Kong and Macao Special Administrative Regions. We excluded Hubei province because it has been sited that laboratory confirmed cases might have potentially been underreported due to the overloaded healthcare system in the region (9). We also collected individual records on reported confirmed COVID-19 cases, including demographic characteristics, exposure and travel history to Hubei, and key timelines, such as date of symptom onset and exposure timeline. Cases with incomplete data were excluded.

Confirmed patients were defined according to the Diagnosis and Treatment Guidelines for Novel Coronavirus Pneumonia (temporary $5^{\text {th }}$ edition) by the National Health Commission of China (10). According to the definition, a confirmed case is an individual who fulfils the criteria of a suspected case based on a comprehensive analysis of epidemiological history and clinical manifestations, and has one of the following types of pathogenic evidence: (I) positive real-time fluorescent polymerase chain reaction (PCR) of respiratory specimens or blood specimens for the detection of novel coronavirus nucleic acid; or (II) respiratory specimens or blood specimens for viral gene sequencing, which are highly homologous with known novel coronaviruses. No personal identifying information were reported in the data, and informed consent was waived. 
An imported case was defined as a patient who travelled to the region after being exposed to an infection in a different region. History of travel to Hubei was defined as a nonHubei habitual resident having travelled or temporarily lived in Hubei before the onset of illness within 14 days. History of exposure within the family was defined as more than one confirmed case of COVID-19 among family members. Since the outbreak happened during the Chinese Lunar New Year holiday, which is culturally the largest and most important holiday of the year, family members were defined as not only the people living in the same household with the children, but also relatives that may have had contact with the children.

\section{Statistical analysis}

Descriptive statistics, including mean and standard deviation (SD), were calculated for continuous variables, median, and interquartile ranges (IQR) for categorical variables. Chisquare test was applied to estimate the association between categorical variables and age groups. Histograms were constructed to describe the key time-to-event distributions, and mosaic plots were used to visualize the relationships among habitual residence, the history of exposure within a family, and the history of regional exposure.

On Jan 20, 2020, China officially confirmed that SARSCoV-2 could be transmitted from human-to-human and COVID-19 was included in the statutory report of Class B infectious diseases and border health quarantine infectious diseases, which marked the transition from the initial partial control approach in Wuhan, Hubei to the comprehensive nationwide adoption of various containment measures in accordance with the law. We used the date of Feb 3, 2020, which is 14 days (considered as the maximum incubation period) after the incorporation of COVID-19 into class $B$ infectious diseases of the law of the People's Republic of China on the Prevention and Treatment of Infectious Diseases and national wide preventive and control measures were implemented, to divide the epidemic into two time periods. The first period ran from Jan 11 to Feb 3, and the second period ran from Feb 4 to Feb 14. We present the results as odds ratios for all children, and for subgroups of children, categorized as those aged $0-5,6-11$, and 12-17 years old, which represented preschool children, schoolchildren, and middle school students respectively.

All data analysis was conducted using R version 3.6.1 (11). The study was reported in accordance with the STrengthening the Reporting of OBservational studies in Epidemiology
(STROBE) recommendations.

\section{Results}

The first child confirmed COVID-19 outside Hubei was diagnosed in Guangdong province on Jan 20, 2020. We collected data from 376 confirmed COVID-19 infections among children from 149 cities in 26 provinces and regions outside Hubei, reported from Jan 20 to Feb 13, 2020 (Figure 1). Of them, $117(31.1 \%)$ were aged 0 to 5 years old, 130 (34.6\%) 6 to 11 years old, and 129 (34.3\%) 12 to 17 years old. Among all the children, 204 (54.7\%) were male and 169 $(45.3 \%)$ were female. The proportion of girls was higher than boys in pre-school aged children (aged 0 to 5 years), but lower among school-age children (Table 1). Ninetyseven $(25.8 \%)$ children were habitual residents of Hubei and were temporarily visiting or living in another region at the time of diagnosis. The proportion of Hubei residents was slightly lower in children aged $12-17$ than in the other age groups.

The median duration from illness onset to first health facility visit was 0.5 day (IQR: $0-2$ ), and the median duration from first health facility visit to diagnosis was 2 days (IQR: 1-3). The median number of days from illness onset to diagnosis was 3 days (IQR: 2-5) (Figure 2). Of the 376 children, $205(54.5 \%)$ had a history of exposure in Hubei, and $156(41.5 \%)$ were locally infected. Only 15 (4\%) children with COVID-19 were exposed in regions other than Hubei or the region of diagnosis. Only 13 children had both the exact time of exposure and the time of illness onset, with a median incubation period of 11 (IQR: 7-13) days. The duration from the end of exposure to illness onset could be calculated for 87 children (median 7 days, IQR: 4-11), while the duration from the exposure onset to illness onset for 40 children was reported (median 13 days, IQR: 9-19) (Figure 3). A total of 233 (62.0\%) children reported family exposure. The remaining $143(38.0 \%)$ children were the first infected individuals among their family members.

Figure 4 shows the mosaic plots of the relationship between habitual residence, the history of family exposure, and the region of exposure (Figure 4). For each mosaic plot, the right-hand side represents children originally from Hubei who was diagnosed with COVID-19 in other regions. The top-left cells represent non-Hubei resident children who were exposed in Hubei and later diagnosed with COVID-19 after leaving Hubei (i.e., imported cases from Hubei). The middle-left cells represent locally infected children, and bottom-left cells children exposed in 

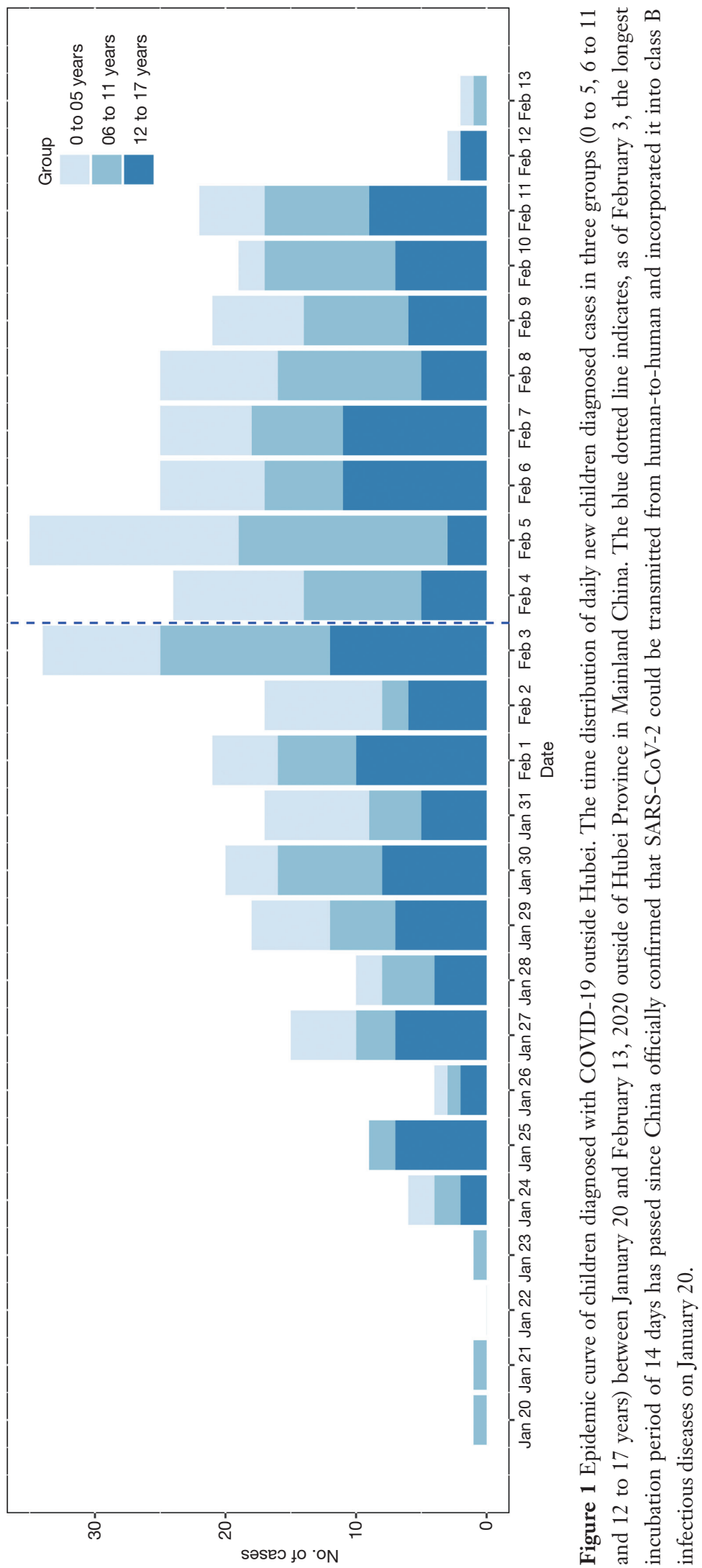
Table 1 Demographic and epidemiologic characteristics in children under 18 years of age with COVID-19 diagnosed outside of Hubei, China

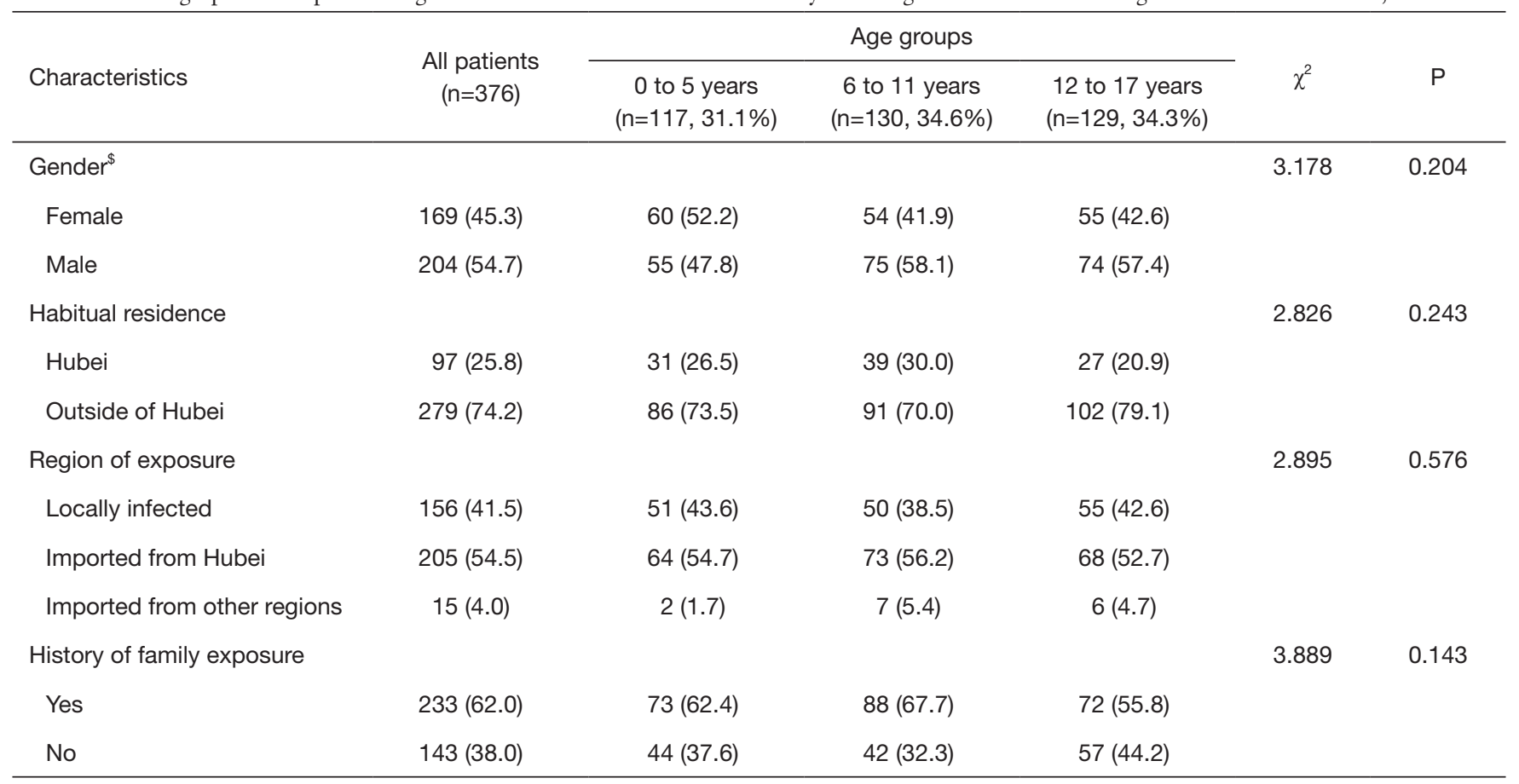

${ }^{\$}$ Data regarding gender were missing for 3 patients.

other regions than Hubei or the region of diagnosis. From the comparison of the two mosaic plots, it can be seen that in period 2, the proportion of imported cases from Hubei decreased, while the proportion of confirmed cases who were exposed via a family member increased. The Mosaic plots proved that the restriction policy was able to control the epidemic and to reduce the outside-family infections rate of children.

We found that children diagnosed during the first period had lower odds of exposure outside of the family than those diagnosed in the second period [odds ratio $(\mathrm{OR})=0.594$, $95 \%$ confidence interval (CI): $0.391,0.904]$. The same trend was seen in the sub-group analyses of the three age groups. The OR of exposure outside of the family was lowest among the children who were exposed in Hubei and were aged 0 to 5 years old $(0.234,95 \%$ CI: $0.082,0.720)$ (Table 2).

\section{Discussion}

Our research reveals that children of different age group and gender are all susceptible to SARS-CoV-2. However, it seems there is a slight difference in the constituent ratio of boys and girls (54.7\% vs. $45.3 \%$ ) outside Hubei, which are consistent with the findings (56.6\% vs. $43.4 \%)$ in mainland China (12). Although the COVID-19 appears to be a disease that predominantly affects the elderly people, more and more child's COVID-19 cases reported in the various province in mainland China after the first case diagnosed outside Hubei on January 20, and eventually changed the preliminary opinion that children were insusceptible to SARS-CoV-2. The results of an epidemiological investigation in Guangdong province showed that children appeared to be as susceptible as adults to SARS-CoV-2, as well as researches improved that the younger people seemed to be inclined to be asymptomatic (13). Therefore, COVID-19 among children may be underestimated. Actually there have been far more COVID-19 confirmed cases in children (965 under 20 years old, reported until Feb 11, 2020), compared with confirmed Severe Acute Respiratory Syndrome (SARS) and Middle East Respiratory Syndrome (MERS) cases in children (14-16). Considering the unavailability of specific effective antiviral therapies or vaccine for the disease, COVID-19 among children should still be highly concerned. In response to the previously unknown novel coronavirus, China has carried out ambitious and aggressive disease containment measures, such as treating confirmed cases with symptomatic support, 

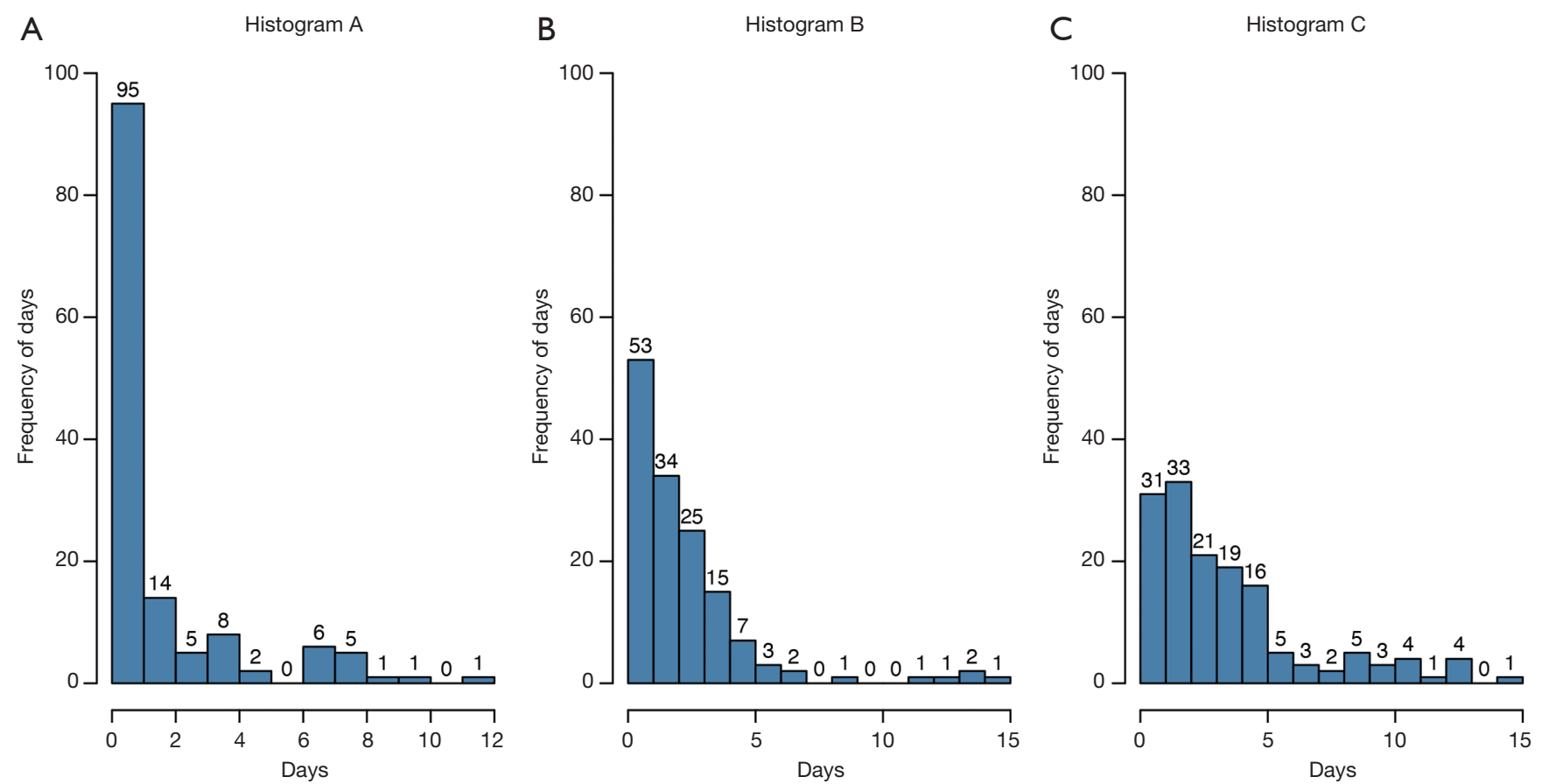

Figure 2 Key time-to-event distributions. (A) The duration from illness onset to first health facility visit; (B) the duration from first health facility visit to diagnosis; (C) the duration from illness onset to diagnosis.

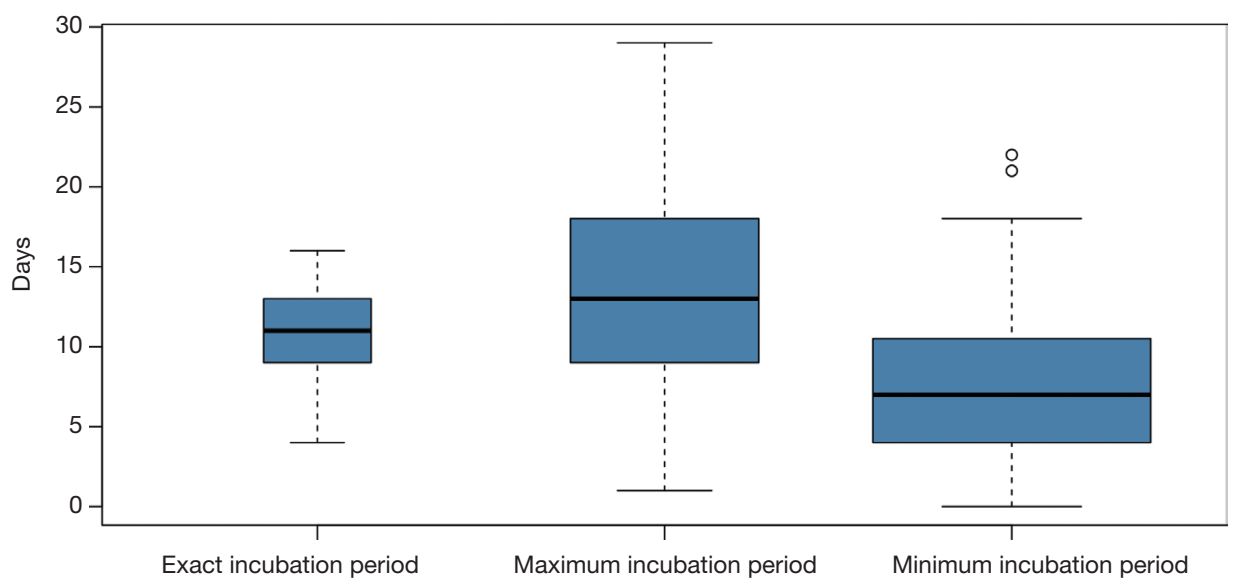

Figure 3 Reported incubation period from children under 18 years old with COVID-19 diagnosed outside of Hubei, China. Maximum period is calculated from the start of exposure, and minimum period from the end of exposure.

quarantining close contacts, putting travelers from Hubei under medical observation, and extending the RT-PCR test. Additionally, the government extended both the Chinese Lunar New Year holiday and winter vacation, and implemented traffic and travel restrictions to control transportation capacity in order to limit population movements (17). As the Figure 1 shows, the number of cases aged 17 years and below had been peaked on Feb 5 and then declined since Feb 6, 14 days after the lockdown of Wuhan city, epicenter of China.

In age stratified analysis, the number of confirmed cases exposed outside family decreased among all age groups after Feb 4, 2020, despite the increasing number of total confirmed cases (Table 2), which suggests that containment 

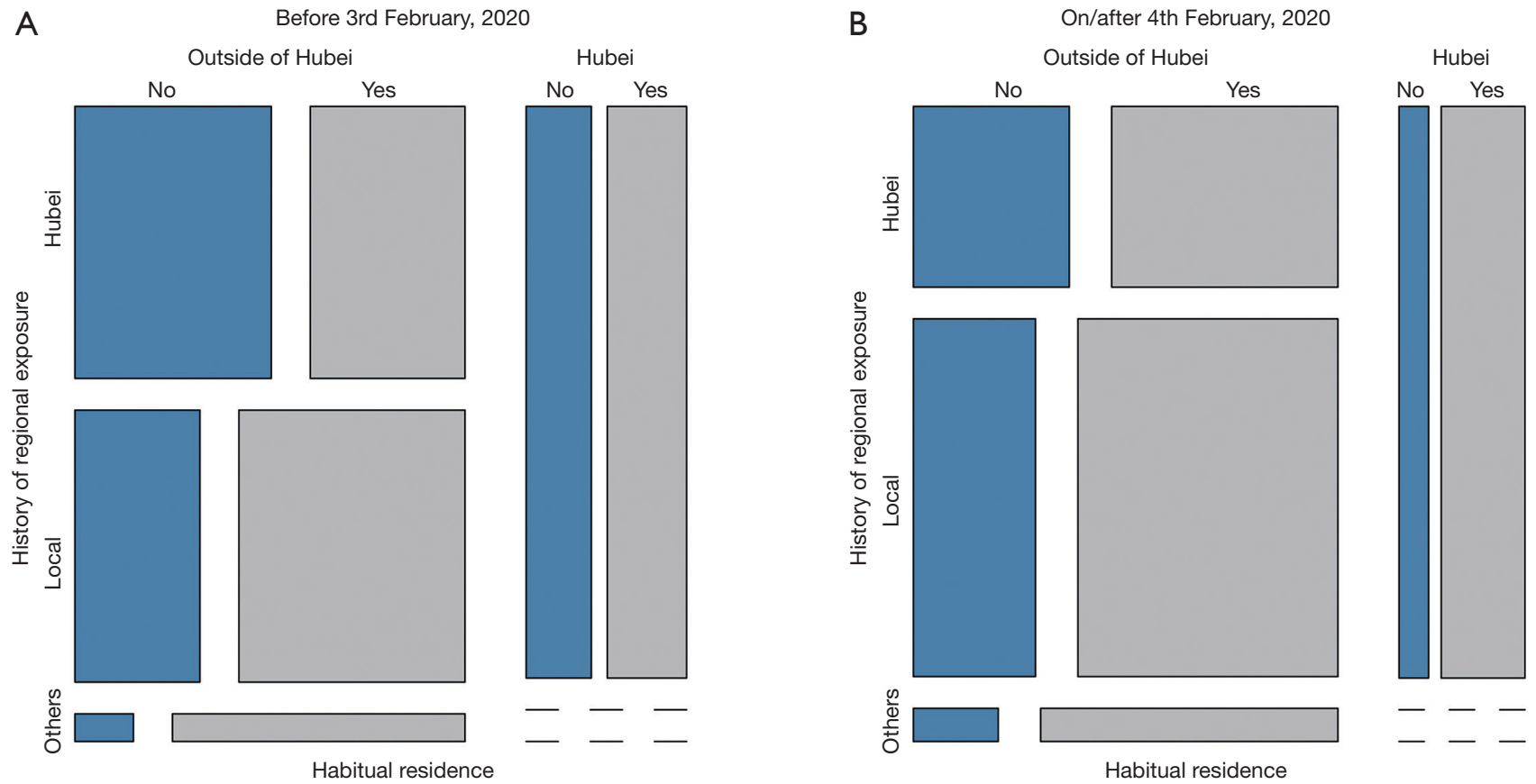

Figure 4 Mosaic plots of family exposure history, region of exposure and habitual residence in confirmed cases under 18 years of age in China outside of Hubei in period 1 (A) and period 2 (B). Grey cells represent exposure within family, dark blue cells represent exposure outside family. Horizontal axis represents the region of habitual residence (right: Hubei; left: other regions outside of Hubei in mainland China). Vertical axis represents the region of exposure (up: imported from Hubei; middle: locally infected; bottom: imported from other regions.

measures have reduced the risk of exposure outside family for children from all age groups. The children aged 0 to 5 years had the lowest odds of being exposed outside of the family (OR $=0.420,95 \% \mathrm{CI}: 0.196$ to 0.904$)$ compared to the other age groups, which indicated that the containment measures were more effective for preschool children who had less active social life than school-age children. Of all confirmed cases with a history of exposure within family, however, only confirmed cases among children aged 12 to 17 years decreased. In light of the report presented by Chinese Center for Disease Control and Prevention, the considerable transmission of COVID-19 was occurring among close contacts, and $64 \%$ of clusters documented have been within familial households (18). Household transmission remains a key route of transmission for children, most of whom were infected by adults or family members (19). In China, children aged 0 to 5 years old usually spend almost all of their time with their family members; therefore, exposure within family would not be greatly affected by containment measures, but might increase due to the gathering of family members during the Spring Festival. Children aged 6 to 11 years old also spent most of their time staying at home or visiting relatives and friends because of the Spring Festival and winter vacation. However, children aged 12 to 17 years old who were reportedly at lower odds of exposure within the family might be related to better compliance and effectiveness of prevention measures, such as wearing a mask and washing their hands. As the containment measures might reduce outside exposures and simultaneously increase infections within family. The negative impact of the restrictive policy on children was to increase the risk of infection within the family. Therefore, we recommend that policy makers should take the family clusters infected children into account and promote sanitation and hygiene practices to protect children, especially the younger ones from infection.

We also found the average time from the onset of illness to the first visit to a medical institution was 0.5 days which shown Chinese parents respond so quickly to symptoms in their children. First, during the early phase of COVID-19 epidemic, there were enough medical resources in nonHubei area of mainland China, which could provide timely 


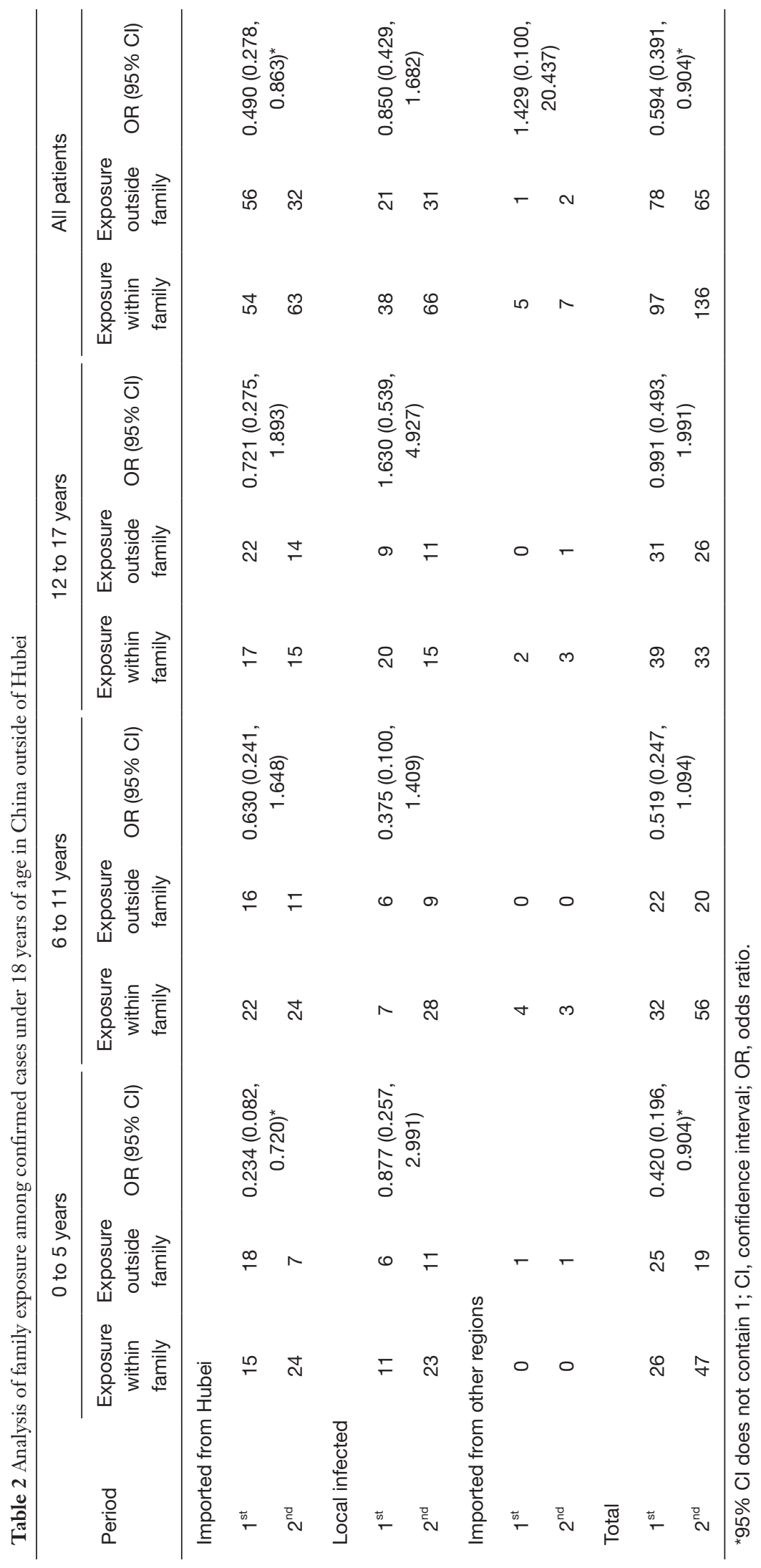


medical test and treatment to the public. Second, China's prevention and control policy required close contacts of confirmed cases to be quarantined immediately for medical observation, so children who developed symptoms during quarantine were able to seek medical attention immediately. Third, in the health concept of Chinese families, parents pay more attention to their children's health than to themselves, especially during the special period of the epidemic. Even if the symptoms of children infected with COVID-19 were less obvious than those in adults, parents would still remain highly vigilant and seek medical attention quickly.

In this study, slightly over half of the cases in children were exposed in Hubei, and about one-quarter of the children diagnosed were Hubei residents temporarily travelling or living in other regions. Compared the proportions of confirmed cases with different regional exposures of two periods, we believe that the containment measures adopted in various places are effective in reducing the exposure of children to COVID-19 outside their families and have played a significant role in preventing the spread of SARS-nCoV-2, especially considering the largescale population movement during the Chinese Lunar New Year holiday and the epicenter of Wuhan, which is one of the four major railway transportation hubs in China. The effect was particularly clear among imported cases from Hubei, which may have been impacted by the control measures taken by various regions, such as the policy of quarantining all the people with history of exposure in Hubei, thus increasing the detection rate of children who were asymptomatic or mild. However, the odds of children exposed locally did not differ between the two time periods, indicating that containment measures may not have an effect on decreasing locally transmitted cases.

At the early stage of the outbreak, there were various factors affecting the development of ongoing epidemic and the effects of the prevention and control measures. Through a special view of certain age groups in different regions, our study provided a new approach to reduce the influence of confounders in research and to assess the effectiveness of the implementation of disease prevention a control policy.

Our study has some limitations. First, this is a crosssectional study, the causality could not be assessed. Additionally, the data used was from publicly available reports and had incomplete epidemiological history information. Therefore, we excluded these cases from certain regions, such as in Beijing. Our findings may not be generalizable to the whole country and may also underestimate the effect of exposure within family. Second, our study shows that the incubation period of COVID-19 for children is longer than the general population (20-22). The incubation period was estimated in only 113 children with documented information, though the smaller sample size might have inevitably affected this estimate. Third, the limited information of confirmed cases in children also limited the ability to do more detailed, stratified analyses to evaluate the effect of containment measures in other aspects. A continuation of data collection and analysis are necessary to further bolster the findings of this study.

\section{Conclusions}

Our study on the children infected with COVID-19 as well as their exposure within family provide evidence that the implementation of containment measures was effective in reducing the odds of exposure outside of the family, especially for preschool children. Continuation of these efforts, coupled with tailored prevention and health education messaging for younger aged children, may help to reduce the transmission of COVID-19 among children until other therapeutic interventions or vaccines are available.

\section{Acknowledgments}

Funding: This work was supported by the National Natural Science Foundation of China (grant 81903406) and the Alibaba (China)-Hong Kong Polytechnic University Collaborative Research grant. The funders were not involved in the research and preparation of the article, including study design; collection, analysis, and interpretation of data; writing of the article; or in the decision to submit it for publication.

\section{Footnote}

Reporting Checklist: The authors have completed the STROBE reporting checklist. Available at http://dx.doi. org/10.21037/tp-20-214

Conflicts of Interests: All authors have completed the ICMJE uniform disclosure form (available at http://dx.doi. org/10.21037/tp-20-214). The authors have no conflict of interest to declare.

Ethical Statement: The authors are accountable for all aspects of the work in ensuring that questions related 
to the accuracy or integrity of any part of the work are appropriately investigated and resolved. The ethical approval or individual consent was not applicable. Data were analyzed at aggregate level and no participants were contacted. The study was performed in accordance with the Declaration of Helsinki (2013 revision).

Open Access Statement: This is an Open Access article distributed in accordance with the Creative Commons Attribution-NonCommercial-NoDerivs 4.0 International License (CC BY-NC-ND 4.0), which permits the noncommercial replication and distribution of the article with the strict proviso that no changes or edits are made and the original work is properly cited (including links to both the formal publication through the relevant DOI and the license). See: https://creativecommons.org/licenses/by-nc-nd/4.0/.

\section{References}

1. World Health Organization. Coronavirus disease 2019 (COVID-19) Situation Report - 41. Available online: https://www.who.int/docs/default-source/coronaviruse/ situation-reports/20200301-sitrep-41-covid-19. pdf?sfvrsn=6768306d_2. (accessed 2 Mar 2020).

2. Phelan AL, Katz R, Gostin LO. The Novel Coronavirus Originating in Wuhan, China: Challenges for Global Health Governance. JAMA 2020;323:709-10.

3. World Health Organization. Report of the WHO-China Joint Mission on Coronavirus Disease 2019 (COVID-19). Available online: https://www.who.int/docs/default-source/ coronaviruse/who-china-joint-mission-on-covid-19-finalreport.pdf. (accessed 1 Mar 2020).

4. Chan JFW, Yuan S, Kok KH, et al. A familial cluster of pneumonia associated with the 2019 novel coronavirus indicating person-to-person transmission: a study of a family cluster. Lancet 2020;395:514-23.

5. Wei M, Yuan J, Liu Y, et al. Novel Coronavirus Infection in Hospitalized Infants Under 1 Year of Age in China. JAMA 2020;323:1313-4.

6. $\mathrm{Xu} X W, \mathrm{Wu} \mathrm{XX}$, Jiang $\mathrm{XG}$, et al. Clinical findings in a group of patients infected with the 2019 novel coronavirus (SARS-Cov-2) outside of Wuhan, China: retrospective case series. BMJ 2020;368:m606.

7. Yu P, Zhu J, Zhang Z, et al. A familial cluster of infection associated with the 2019 novel coronavirus indicating potential person-to-person transmission during the incubation period. J Infect Dis 2020;221:1757-61.

8. The Novel Coronavirus Pneumonia Emergency
Response Epidemiology Team of Chinese Center for Disease Control and Prevention. The epidemiological characteristics of an outbreak of 2019 novel coronavirus diseases (COVID-19) in China (in Chinese). Zhonghua Liu Xing Bing Xue Za Zhi 2020;41:145-51.

9. Wu JT, Leung K, Leung GM. Nowcasting and forecasting the potential domestic and international spread of the 2019-nCoV outbreak originating in Wuhan, China: a modelling study. Lancet 2020;395:689-97.

10. National Health Commission of China. Diagnosis \& Treatment Guideline for Novel Coronavirus Pneumonia (temporary 5th edition) (in Chinese). Available online: http://www.nhc.gov.cn/yzygj/s7653p/202002/3b09b894ac 9b4204a79db5b8912d4440.shtml. (accessed 15 Feb 2020).

11. R Core Team (2019). R: A language and environment for statistical computing. R Foundation for Statistical Computing, Vienna, Austria. Available online: http://www. R-project.org/.

12. Dong $\mathrm{Y}, \mathrm{Mo} \mathrm{X}, \mathrm{Hu} \mathrm{Y}$, et al. Epidemiological Characteristics of 2143 Pediatric Patients With 2019 Coronavirus Disease in China. Pediatrics 2020;145:e20200702.

13. Bi Q, Wu Y, Mei S, et al. Epidemiology and transmission of COVID-19 in 391 cases and 1286 of their close contacts in Shenzhen, China: a retrospective cohort study. Lancet Infect Dis 2020;20:911-9.

14. Deng SQ, Peng HJ. Characteristics of and Public Health Responses to the Coronavirus Disease 2019 Outbreak in China. J Clin Med 2020;9:575.

15. McCloskey B, Heymann DL. SARS to novel coronavirus - old lessons and new lessons. Epidemiol Infect 2020;148:e22.

16. Wu Z, McGoogan JM. Characteristics of and Important Lessons From the Coronavirus Disease 2019 (COVID-19) Outbreak in China: Summary of a Report of 72314 Cases From the Chinese Center for Disease Control and Prevention. JAMA 2020;323:1239-42.

17. Wilder-Smith A, Freedman DO. Isolation, quarantine, social distancing and community containment: pivotal role for old-style public health measures in the novel coronavirus (2019-nCoV) outbreak. J Travel Med 2020;27:taaa020.

18. Chinese Center for Disease Control and Prevention. Report of the WHO-China Joint Mission on Coronavirus Disease 2019 (COVID-19) 2020. Available online: https:// www.who.int/docs/default-source/coronaviruse/whochina-joint-mission-on-covid-19-final-report.pdf. March $2,2020$.

19. Xu X, Liu X, Wang L, et al. Household transmissions 
of SARS-CoV-2 in the time of unprecedented travel lockdown in China. medRxiv 2020;2020.03.02.20029868.

20. Guan WJ, Ni ZY, Hu Y, et al. Clinical Characteristics of Coronavirus Disease 2019 in China. N Engl J Med 2020;382:1708-20.

21. Huang C, Wang Y, Li X, et al. Clinical features of patients infected with 2019 novel coronavirus in Wuhan, China. Lancet 2020;395:497-506.

22. Li Q, Guan X, Wu P, et al. Early Transmission Dynamics in Wuhan, China, of Novel Coronavirus-Infected Pneumonia. N Engl J Med 2020;382:1199-207.

Cite this article as: Yang S, Feng X, Du P, He D, Estill J, Yang L, Wen C, Luo J, Wang X, Lu L, Li T, Tang X, Li W, Xu H, Liu E, Cao P, Chen Y; on behalf of COVID-19 evidencebased research working group. Family exposure and the impact of containment measures to children with coronavirus disease 2019 outside Hubei, China: a cross-sectional study. Transl Pediatr 2021;10(1):92-102. doi: 10.21037/tp-20-214 